\title{
Über eine Abbildung der Ebene auf eine gewisse Kummer'sche Fläche.
}

\author{
Von Jan de Vries in Utrecht.
}

1. Es sei $A B C$ ein Dreieck, $P$ ein Punkt seiner Ebene. Bezeichnet man die Entfernungen $A P, B P, C P$ beziehungsweise mit $x, y, z$, so besteht zwischen diesen tripolaren Coordinaten und den Seiten $a, b, c$ die bekannte Gleichung

$$
\begin{gathered}
a^{2} x^{4}+b^{2} y^{4}+c^{2} z^{4}-\gamma^{2} x^{2} y^{2}-a^{2} y^{2} z^{2}-\beta^{2} z^{2} x^{2}-a^{2} \alpha^{2} x^{2}- \\
-b^{2} \beta^{2} y^{2}-c^{2} \gamma^{2} z^{2}+a^{2} b^{2} c^{2}=0,
\end{gathered}
$$

wo

$$
\alpha^{2}=b^{2}+c^{2}-a^{2}, \quad \beta^{2}=a^{2}+c^{2}-b^{2}, \quad \gamma^{2}+a^{2}+b^{2}-c^{2}
$$

ist. Betrachtet man $x, y, z$ als die orthogonalen Coordinaten eines Raumpunktes $P^{\prime}$, so stellt obige Gleichung eine biquadratische Fläche $F_{4}$ dar.

Wird jedem Punkte $P$ der Ebene sein Bildpunkt $P^{\prime}$ zugeordnet, so erhält man demnach eine Abbildung der reellen Punkte der Ebene auf den positiven Octanten der Fläche $F_{4}$.

Die Coordinatenebene $z=0$ durchsetzt $F_{4}$ in zwei Kegelschnitten mit den Gleichungen

$$
\begin{aligned}
& 2 a^{2} x^{2}-\left(\gamma^{2}-\delta^{2} i\right) y^{2}=a^{2}\left(\alpha^{2}+\delta^{2} i\right) \\
& 2 a^{2} x^{2}-\left(\gamma^{2}+\delta^{2} i\right) y^{2}=a^{2}\left(a^{2}-\delta^{2} i\right),
\end{aligned}
$$

wo $i=\sqrt{-1}$ und

$$
\delta^{4}=2 a^{2} b^{2}+2 b^{2} c^{2}+2 c^{2} a^{2}-a^{4}-b^{4}-c^{4}
$$

ist. Diese imaginären Curven schneiden sich in den reellen Punkten

$$
x^{2}=b^{2}, \quad y^{2}=a^{2}, \quad z=0 .
$$

Durch eine einfache Rechnung ergibt sich, dass sie Doppelpunkte der Fläche sind. 
Dies gilt offenbar auch für die weiteren Punkte

$$
\begin{aligned}
& x=0, \quad y^{2}=c^{2}, \quad z^{2}=b^{2} \\
& y=0, \quad x^{2}=c^{2}, \quad z^{2}=a^{2} .
\end{aligned}
$$

Aufer diesen zwölf Doppelpunkten gibt es noch vier Doppelpunkte in der unendlich fernen Ebene. Sie werden aus $O$ projiciert durch die Geraden

$$
x^{2}=y^{2}=z^{2} .
$$

Auch die unendlich ferne Ebene trifft $F_{4}$ in zwei imaginären Kegelschnitten, welche durch die Kegelflächen

$$
2 a^{2} x^{2}-\left(\gamma^{2} \pm \delta^{2} i\right) y^{2}-\left(\beta^{2} \mp \delta^{2} i\right) z^{2}=0
$$

projiciert werden.

Wie leicht ersichtlich, liegen die sechszehn Doppelpunkte von $F_{4}$ zu je sechs in den sechszehn Ebenen

$$
\begin{array}{cc}
a x \pm b y \pm c z=0, & x \pm y \pm c=0, \\
x \pm z \pm b=0, & y \pm z \pm a=0 .
\end{array}
$$

Nachdem diese Ebenen zu vieren durch den Punkt $O$ und die unendlich fernen Punkte von $O X, O Y, O Z$ gehen, ist $F_{4}$ eine Kummer'sche Fläche von der Art, welche Cayley als Tetraedroid bezeichnet hat.

2. Aus der Identität

$$
\begin{gathered}
4 a^{2} b^{2} c^{2} F_{4}+\delta^{4}(a x+b y+c z)(a x+b y-c z)(a x-b y+c z)(-a x+b y+c z) \equiv \\
\equiv a^{2} a^{2} x^{2}+b^{2} \beta^{2} y^{2}+c^{2} \gamma^{2} z^{2}-2 a^{2} b^{2} c^{2}
\end{gathered}
$$

erhellt, dass die vier Ebenen

$$
a x \pm b y \pm c z=0
$$

das Tetraedroid in vier Kegelschnitten berühren, welche auf der quadratischen Fläche

$$
a^{2} \alpha^{2} x^{2}+b^{2} \beta^{2} y^{2}+c^{2} \gamma^{2} z^{2}=2 a^{2} b^{2} c^{2}
$$

liegen.

Hieraus ergibt sich beiläufig, dass die letzte Gleichung, in tripolaren Coordinaten den Umkreis des Dreieckes darstellt. ${ }^{1}$ )

Es sind nämlich

$$
a x+b y=c z, \quad a x+c z=b y, \quad b y+c z=a x
$$

dem Ptolemaeischen Satze zufolge die tripolaren Gleichungen der drei Bogen $A B, A C, B C$ jenes Kreises.

1) J. de Vries, Recherches sur les coordonnées multipolaires, Archives Teyler, série II, tome V, p. 148. (Seite 50 des Sonderabdrucks). 
Analog erhellt aus der Beziehung

$$
\begin{gathered}
4 c^{2} F_{4}+\delta^{4}(x+y+c)(x+y-c)(x-y+c)(-x+y+c) \equiv \\
\equiv\left(\beta^{2} x^{2}+\alpha^{2} y^{2}-2 c^{2} z^{2}+\gamma^{2} c^{2}\right)^{2} .
\end{gathered}
$$

Dass die vier singulären Tangentialebenen

$$
x \pm y \pm c=0
$$

in Kegelschnitten der quadratischen Fläche

berühren.

$$
\beta^{2} x^{2}+\alpha^{2} y^{2}-2 c^{2} z^{2}+y^{2} c^{2}=0
$$

3. Wenn $A B C$ ein gleichseitiges Dreieck ist, wird die Gleichung des Tetraedroids

$$
O_{4} \equiv x^{4}+y^{4}+z^{4}-x^{2} y^{2}-y^{2} z^{2}-z^{2} x^{2}-a^{2} x^{2}-a^{2} y^{2}-a^{2} z^{2}+a^{4}=0 \text {. }
$$

Alsdann sind die sechzehn Knoten die Kantenmitten und die unendlich fernen Punkte der Hauptdiagonalen eines Würfels. Sie liegen demnach zu vieren in sechzehn Ebenen, von denen sechs mit den Würfelflächen, sechs mit Diagonalebenen und drei mit den Coordinatenebenen zusammenfallen, während die letzte Ebene unendlich fern liegt. Die Knotenpunkte gehören somit nicht nur einer Configuration $\left(16^{6}, 16_{6}\right)$, sondern auch einer $\left(16^{4}, 16_{4}\right)$ an. $\left.{ }^{1}\right)$ Aus den identischen Relationen

$$
\begin{aligned}
& 4 O_{4}+3(x+y+z)(x+y-z)(x-y+z)(-x+y+z) \equiv\left(x^{2}+y^{2}+z^{2}-2 a^{2}\right)^{2}, \\
& 4 O_{4}+3(x+y+a)(x+y-a)(x-y+a)(-x+y+a) \equiv\left(x^{2}+y^{2}-2 z^{2}+a^{2}\right)^{2}, \\
& O_{4}+(x+y+a)(x+y-a)(x-y+z)(-x+y+z) \equiv\left(x y+z^{2}-a^{2}\right)^{2}, \\
& O_{4}+(x+y+z)(x+y-z)(x-y+a)(-x+y+a) \equiv\left(x y-z^{2}+a^{2}\right)^{2}, \\
& O_{4}+(x+y+a)(x-y+a)(x+z-a)(-x+z+a) \equiv\left(y^{2}-z^{2}-a x\right)^{2}, \\
& O_{4}+(x+y-a)(-x+y+a)(x+z+a)(x-z+a) \equiv\left(y^{2}-z^{2}+a x\right)^{2},
\end{aligned}
$$

und den analogen Beziehungen erhält man die Gleichungen von sechzehn quadratischen Flächen, welche je zwölf Knoten und vier von den sechzehn Kegelschnitten enthalten, in denen $O_{4}$ von den singulären Tangentialebenen berïhrt wird.

Wenn der Knotenpunkt $x=a, y=a, z=0$ durch das Symbol $\left(-\frac{1}{+}+0\right)$, der auf $x=y=z$ unendlich fern gelegene Punkt dureh $(+++)$ bezeichnet wird, u. s. w., so ergibt sich für die Vertheilung der Knoten auf den singulären Tangentialebenen, beziehungsweise den in ihnen liegenden Kegelschnitten, die Tabelle:

1) Hierzu wolle man vergleichen: J. de Vries, Über räumliche Configurationen, welche sich aus den regelmäßigen Polyedern herleiten lassen, Wiener Sitzungsberichte, Bd. 100, S. 822-842, and E. Hess, Beiträge zur Theorie der räumlichen Configurationen, Nova Acta der $k$. Leop. Carol. deutschen Akad. der Naturforscher, Bd. LV, s. 154. 


$$
\begin{aligned}
& x+y+z=0 \mid+-0,-+0,+0-,-0+, 0+-, 0-+ \\
& x+y-z=0+-0,-+0,+0+,-0-, 0++, 0-- \\
& x-y+z=0++0,--0,+0-,-0+, 0++, 0-\cdots \\
& -x+y+z=0++0,--0,+0+,-0-, 0+-, 0-+ \\
& x+y+a=0-0+,-0-, 0-+, 0--,+\cdots+,-++ \\
& x+y-a=0+0+,+0-, 0++, 0+-,+-+,-++ \\
& x-y+a=0-0+,-0-, 0++, 0+-,+++,-\cdots+ \\
& -x+y+a=0+0+,+0-, 0-+, 0--,+++,--+ \\
& x+z+a=00+-, 0--,-+0,--0,-++,-\cdots+ \\
& x+z-a=00++, 0-+,++0,+-0,-++,-\cdots+ \\
& x-z+a=00++, 0-+,-+0,--0,+++,+-+ \\
& -x+z+a=0 \quad 0+-, 0--,++0,+-0,+++,+-+ \\
& y+z+a=0+0-,-0-,+-0,--0,+-+,-\cdots+ \\
& y+z-a=0+0+,-0+,++0,-+0,+\cdots+,--+ \\
& y-z+a=0+0+,-0+,+-0,--0,+++,-++ \\
& -y+z+a=01+0-,-0-,++0,-+0,+++,-+1
\end{aligned}
$$

Wie leicht ersichtlich, sind die in dem positiven Octanten enthaltenen Segmente jener Kegelschnitte, beziehungsweise die Abbildungen der Bogen $A B, B C, C A$ des Umkreises, sowie der Seiten des Dreieckes $A B C$ und deren Verlängerungen.

Ferner wird der Kreis $x=a$ durch zwei Bogen der Ellipsen

$$
x=a, \quad y^{2} \pm y \approx \sqrt{3}+z^{2}=a^{2}
$$

vertreten, welche in den Knoten $(+0+)$ und $(++0)$ zusammenhängen.

Dem Mittelloth $y=z$ entsprechen offenbar zwei den Hyperbeln

$$
y=z, \quad x^{2}-y^{2} \pm a x \sqrt{3}+a^{2}=0
$$

angehörende Bogen, welche in den Knoten $(0++)$ und $(+++)$ in einander übergehen. 